\title{
OPEN Mid-wavelength infrared avalanche photodetector with AlAsSb/GaSb superlattice
}

\author{
Jiakai Li, Arash Dehzangi, Gail Brown \& Manijeh Razeghi ${ }^{\bowtie}$ \\ In this work, a mid-wavelength infrared separate absorption and multiplication avalanche photodiode \\ (SAM-APD) with $100 \%$ cut-off wavelength of $\sim 5.0 \mu \mathrm{m}$ at $200 \mathrm{~K}$ grown by molecular beam epitaxy \\ was demonstrated. The InAsSb-based SAM-APD device was designed to have electron dominated \\ avalanche mechanism via the band structure engineered multi-quantum well structure based on \\ AlAsSb/GaSb H-structure superlattice and InAsSb material in the multiplication region. The device \\ exhibits a maximum multiplication gain of 29 at $200 \mathrm{~K}$ under -14.7 bias voltage. The maximum \\ multiplication gain value for the MWIR SAM-APD increases from 29 at $200 \mathrm{~K}$ to 121 at $150 \mathrm{~K}$. The \\ electron and hole impact ionization coefficients were derived and the large difference between their \\ value was observed. The carrier ionization ratio for the MWIR SAM-APD device was calculated to \\ be $\sim 0.097$ at $200 \mathrm{~K}$.
}

Mid-wavelength infrared (MWIR) photodetectors which can operate under the low flux conditions are of great interest for long-range military and astronomical applications ${ }^{1,2}$. In most of these applications there is a need to increase the capability of the system to detect light in a low photon flux situation. Therefore, gain-based devices such as heterojunction phototransistors (HPTs) and avalanche photodiodes (APDs) are used to achieve the necessary photoresponse when the incoming photon flux is low ${ }^{3-5}$. Compared with the HPTs, the APDs can amplify weak signals without the relatively more complicated HPT device structure ${ }^{6}$.

For MWIR APD devices, HgCdTe is the state-of-art material system and has been widely used in infrared $\mathrm{APDs}^{7,8}$. However, the HgCdTe-based APDs suffer from drawbacks such as material instability and low fabrication yields $s^{9,10}$. The emerging material system, antimony-based strained layer superlattices (SLS) have drawn lots of attention due to the advantages of high material uniformity, great bandgap tunability and Auger recombination suppression compared with $\mathrm{HgCdTe}$ detectors ${ }^{11-14}$. Recently, the MWIR APDs based on III-V superlattices have been demonstrated ${ }^{15}$. However, their performance, especially the excess noise factor, is limited due to the relatively small difference in the ionization rates for electrons and holes or because both electrons and holes are injected into the multiplication region. The SAM structure can be used to reduce excess noise factor and also enhance the multiplication noise gain through impact-ionization engineering ${ }^{16,17}$.

Due to the great band structure engineering flexibility, an antimonide-based SLS can be used as the barrier layer for a multi-quantum well (MQW) heterostructure when combined with an InAsSb well. This MQW enables using the antimony-based SLS to engineer a large difference in the ionization rates for electrons and holes by designing the band discontinuities between well and barrier to have a large difference between conduction band offset $\left(\Delta E_{c}\right)$ and valence band offset $\left(\Delta E_{v}\right)$. As demonstrated by McIntyre ${ }^{18}$, a large difference in the ionization rates for electrons and holes is essential for a low noise avalanche photodiode. Therefore, it is promising to use the MQW approach, with antimony-based SLS barriers, as the multiplication layer in the APDs. The separate absorption layer used in the SAM-APD is an InAsSb alloy chosen to cover the mid-infrared range at an operating temperature of $150 \mathrm{~K}$.

The MWIR SAM-APD device was grown by molecular beam epitaxy with the multiplication layer consisting of an $\mathrm{AlGaAsSb} / \mathrm{InAs}_{0.9} \mathrm{Sb}_{0.1} \mathrm{MQW}$. The AlGaAsSb barrier layer in the MQW structure was grown as an $\mathrm{AlAs}_{0.1} \mathrm{Sb}_{0.9} / \mathrm{GaSb}$ superlattice, This SLS is called an $\mathrm{H}$-structure superlattice and can be used as an electron barrier layer in some type-II superlattice infrared photodetector designs ${ }^{19}$. For convenience, 'AlGaAsSb' will be used to refer to the AlAsSb/GaSb H-structure superlattice in the rest of discussion. The bandgap energy of the $\mathrm{H}$-structure superlattice was calculated to be around $1 \mathrm{eV}$ at $150 \mathrm{~K}$ by the empirical tight-binding method (ETBM) with $s p s^{*}$ formalism which was modified from previous work ${ }^{20,21}$. The ETBM material parameter sets in the previous work were used ${ }^{21}$. The effective conduction band of the $\mathrm{H}$-structure superlattice moves upward 
(a)

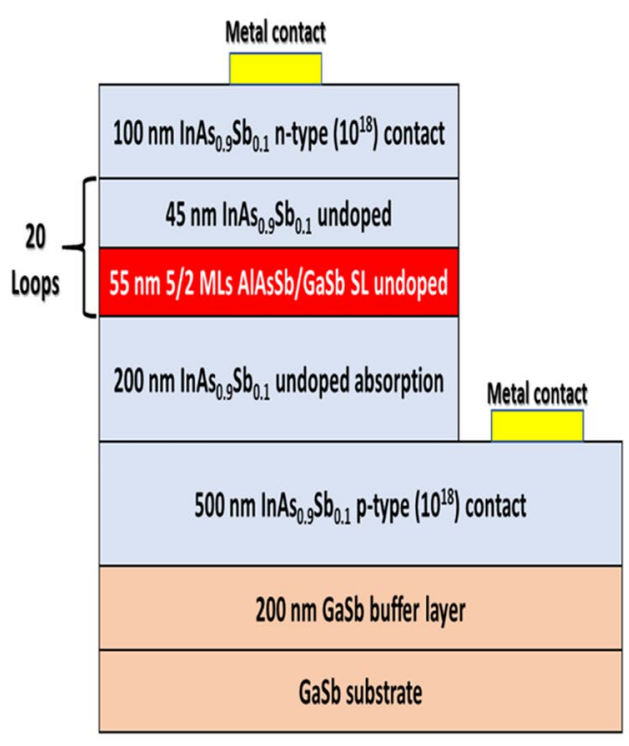

(b)

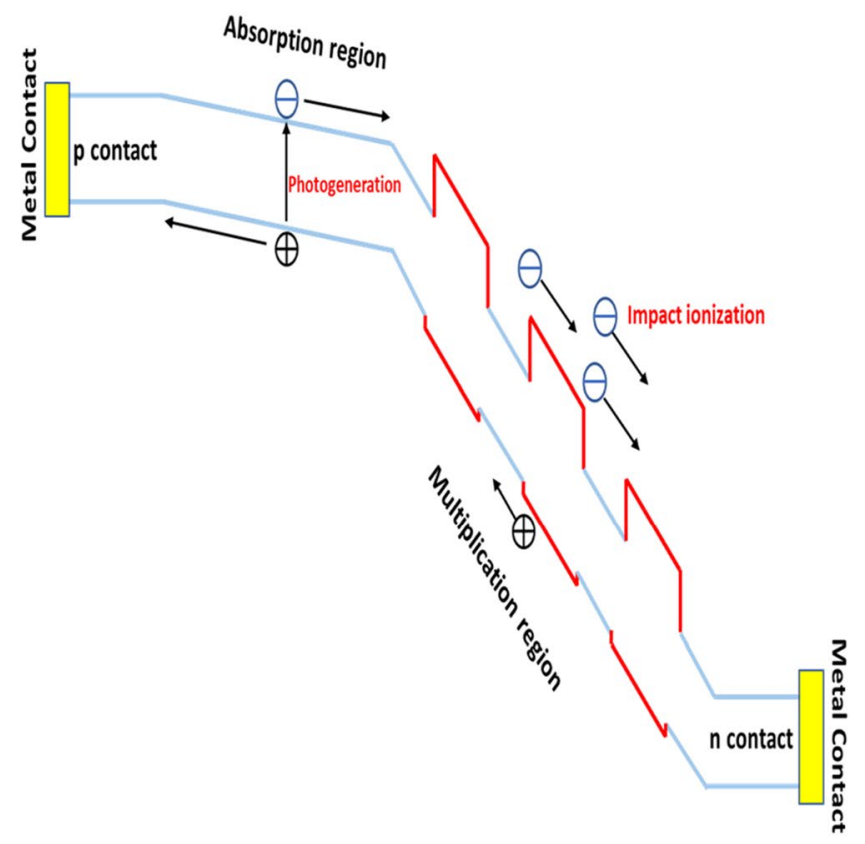

Figure 1. (a) Schematic of the MWIR SAM-APD structure. (b) Schematic band structure of the SAM-APD device under reverse bias.

significantly due to the confinement of the electrons in the GaSb well by the AlAsSb barrier layers. The InAs ${ }_{0.9} \mathrm{Sb}_{0.1}$ bulk material was also used as the absorption layer for the mid-wavelength infrared detection at $150 \mathrm{~K}$. During the growth, all the In $\mathrm{As}_{0.9} \mathrm{Sb}_{0.1}$ layers in the device structure was grown as InAs-InSb binary-binary digital alloy ${ }^{22}$.

The MWIR APD was grown on 2-inch Te-doped n-type $\left(10^{17} \mathrm{~cm}^{-3}\right) \mathrm{GaSb}(100)$ substrate at $385{ }^{\circ} \mathrm{C}$ using an Intevac Modular Gen II molecular beam epitaxy (MBE) and its device structure is shown in Fig. 1(a). The $200 \mathrm{~nm}$ thick GaSb buffer layer and a $500 \mathrm{~nm}$ thick p-contact $\left(10^{18} \mathrm{~cm}^{-3}\right) \operatorname{InAs} s_{0.9} S_{0.1}$ layer was grown. A $200 \mathrm{~nm}$ undoped In $\mathrm{As}_{0.9} \mathrm{Sb}_{0.1}$ absorption layer was grown next. The InAsSb absorption layer was followed by a MQW structure with AlGaAsSb (AlAsSb/GaSb H-structure superlattice) as the barrier layer and $\operatorname{InAs}_{0.9} \mathrm{Sb}_{0.1}$ as the well layer. There are 20 loops of barrier and well layers in this MQW structure. The MQW structure was used as the multiplication layer, as shown in Fig. 1(b). To finish the device, a $100 \mathrm{~nm}$ top n-contact $\left(10^{18} \mathrm{~cm}^{-3}\right) \operatorname{InAs}_{0.9} \mathrm{Sb}_{0.1}$ layer was grown. During growth, silicon and beryllium were used for n-type and p-type dopants, respectively. The schematic band diagram of the SAM-APD device under reverse bias is shown in Fig. 1(b).

After the MBE growth, the material quality was assessed using atomic force microscopy (AFM) and high resolution X-ray diffraction (HR-XRD). As shown in Fig. 2(a), the sample surface exhibits clear atomic steps with a small RMS roughness of $0.162 \mathrm{~nm}$ over $10 \mu \mathrm{m} \times 10 \mu \mathrm{m}$ area. Figure 2(b) shows the XRD scan curve of the MWIR APD sample, where the GaSb substrate, AlAsSb/GaSb H-structure SL and InAsSb layer peaks are marked. The mismatch between the AlAsSb/GaSb H-structure superlattice and the GaSb substrate is $\sim 2500$ ppm, while the InAsSb layer exhibits a negative mismatch of $\sim-1500 \mathrm{ppm}$. The higher-order satellite peaks in the XRD curve are not very strong and clear, which may be caused by the non-uniformity of the superlattice periodicity of AlAsSb/ $\mathrm{GaSb} \mathrm{H}$-structure SL and can be further optimized by reducing the thickness of AlAsSb layers.

The conduction band offset $\left(\Delta E_{c}\right)$ is larger than the valence band offset $\left(\Delta E_{v}\right)$ between the barrier and well in the MQW structure according to the ETBM. As shown in Fig. 1(b), when a hot electron in the AlGaAsSb barrier of the MQW structure, accelerated by the applied bias voltage, enters a InAsSb well, it abruptly gains energy equal to the conduction band edge $\left(\Delta E_{c}\right)$. The net effect is that the electron sees a stronger electric field (increased by $\left.\Delta E_{c}\right)$. Since the electron impact ionization rate in the InAsSb well layer, $\alpha_{\text {InAsSb }}^{\prime}$, increases exponentially with increasing electric field, a large increase in the electron impact ionization rate is expected. When the electron enters the next AlGaAsSb barrier, it sees a field decreased by $\Delta E_{c}$ and thus a reduced electron impact ionization rate in the AlGaAsSb barrier layer ${ }^{23}$. However, since $\alpha_{I n A s S b}^{\prime} \gg \alpha_{A l G a A s S b}^{\prime}$, the exponential dependence on the threshold energy ensures that the average electron impact ionization rate $\alpha:^{24}$

$$
\bar{\alpha}=\left(\alpha_{I n A s S b}^{\prime} L_{I n A s S b}+\alpha_{A l G a A s S b}^{\prime} L_{A l G a A s S b}\right) /\left(L_{I n A s S b}+L_{A l G a A s S b}\right)
$$

is largely increased ( $L$ denotes layer thicknesses). In contrast, the hole ionization rate $\beta$ is not substantially changed by the MQW structure since the valence band discontinuity $\left(\Delta E_{v}\right)$ is much smaller. The holes can flow unhindered across the MQW multiplication layer. Therefore, the hole impact ionization rate $\beta$ in the MQW structure is expected to have a similar value of $\beta$ as in the bulk InAsSb material. In general, the difference between the electron impact ionization rate and the hole impact ionization rate can lead to a large reduction in the $\beta / \alpha$ ratio and lead to the pure or dominant electron-initiated multiplication mechanism in the MWIR APD. 
(a)

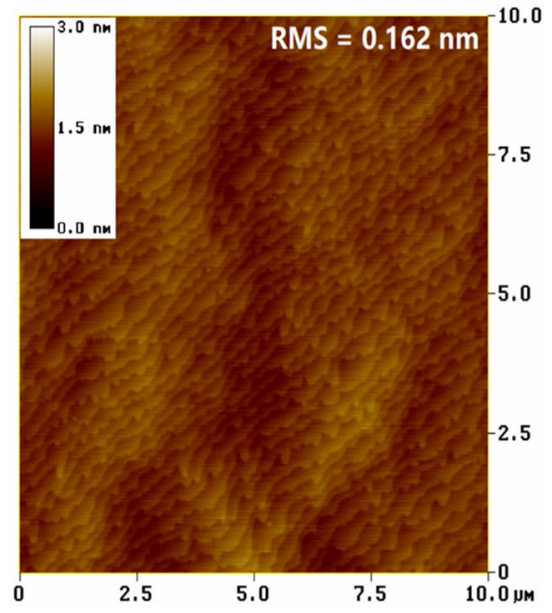

(b)

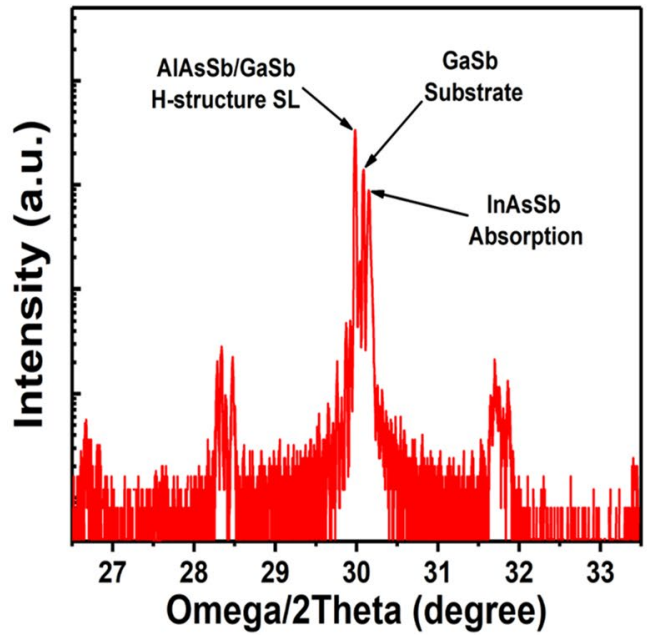

Figure 2. (a) The atomic force microscopy image of the grown MWIR APD sample over $10 \mu \mathrm{m} \times 10 \mu \mathrm{m}$ area. (b) HR-XRD scan curve of the grown APD sample, where peaks of different regions are marked.

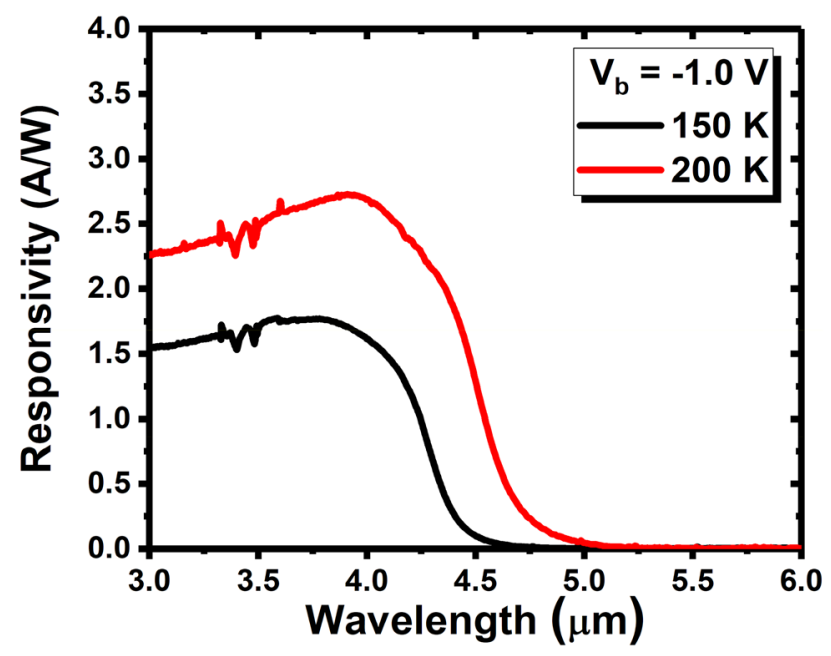

Figure 3. Responsivity spectra of the SAM-APD device under front-side illumination at $150 \mathrm{~K}$ (black) and $200 \mathrm{~K}$ (red) under $-1.0 \mathrm{~V}$ bias voltage.

The grown material was processed into a set of circular photodetectors using conventional photodetector processing ${ }^{25,26}$. An inductive couple plasma reactive ion etching (ICP-RIE) system followed by a citric acid-based isotropic wet etching solution of $\mathrm{C}_{6} \mathrm{H}_{8} \mathrm{O}_{7}: \mathrm{H}_{2} \mathrm{O}_{2}: \mathrm{H}_{3} \mathrm{PO}_{4}: \mathrm{H}_{2} \mathrm{O}$ (5:2:1:20) was used during mesa etching to have smooth sidewalls. No anti-reflection (AR) coating was applied, and the devices were passivated for protection and insulation purposes by $600 \mathrm{~nm} \mathrm{SiO}_{2}$ using plasma-enhanced chemical vapor deposition (PECVD). The test chip was then mounted onto a 68-pin leadless chip carrier (LCC) for electrical and optical characterization. The SAM-APD test chip was loaded into a Janis STVP-100 two chamber liquid helium cryostat station with controlled temperature ranging from 150 to $200 \mathrm{~K}$.

The relative spectral response of the InAsSb-based MWIR SAM-APD was measured at both $150 \mathrm{~K}$ and $200 \mathrm{~K}$ under front-side illumination using a Bruker IFS 66v/S Fourier transform infrared spectrometer (FTIR). A calibrated blackbody source at $1000{ }^{\circ} \mathrm{C}$ was then used to calculate the absolute optical responsivity of the photodiodes $^{27}$. The optical performance of the devices is shown in Fig. 3. At $150 \mathrm{~K}$ and $200 \mathrm{~K}$, the responsivity for the SAM-APD device reaches a peak value of $1.71 \mathrm{~A} / \mathrm{W}$ and $2.72 \mathrm{~A} / \mathrm{W}$ at $3.9 \mu \mathrm{m}$ under $-1.0 \mathrm{~V}$ applied bias, respectively. The device exhibits a $100 \%$ cut-off wavelength of $\sim 4.6 \mu \mathrm{m}$ at $150 \mathrm{~K}$ and $\sim 5.0 \mu \mathrm{m}$ at $200 \mathrm{~K}$.

Current-voltage (I-V) measurements of the SAM-APD sample were carried utilizing an Agilent $4156 \mathrm{c}$ semiconductor parameter analyzer. To study the temperature dependent gain characteristics, the measurement temperature for the MWIR InAsSb APDs was varied from 150 to $250 \mathrm{~K}$. A $633 \mathrm{~nm} \mathrm{He}-\mathrm{Ne}$ laser with an incident power of $5.0 \mathrm{~mW}$ was used to measure the photocurrent and the gain of the APDs. The multiplication gain was calculated by normalizing the photocurrent, i.e. difference between light and dark currents, by the unity-gain 
(a)

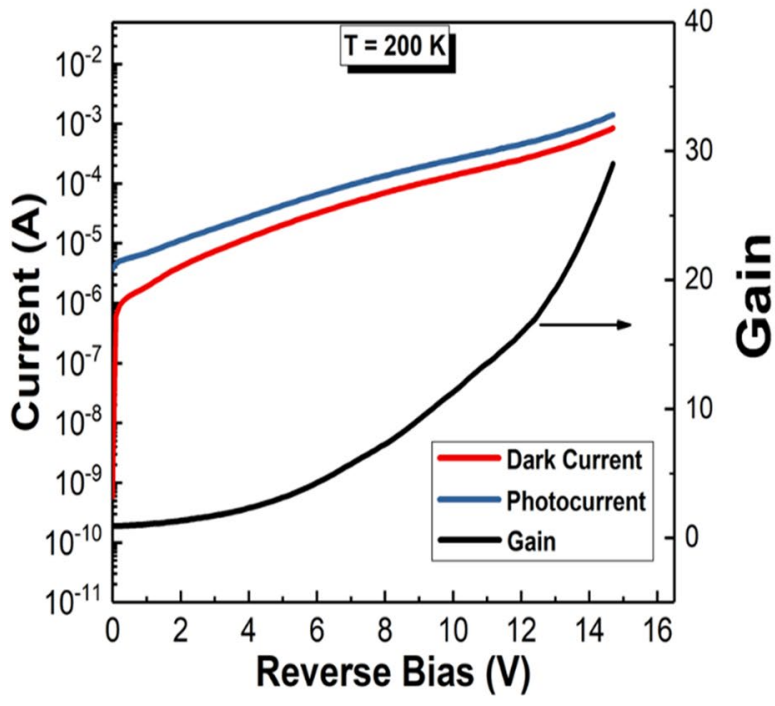

(b)

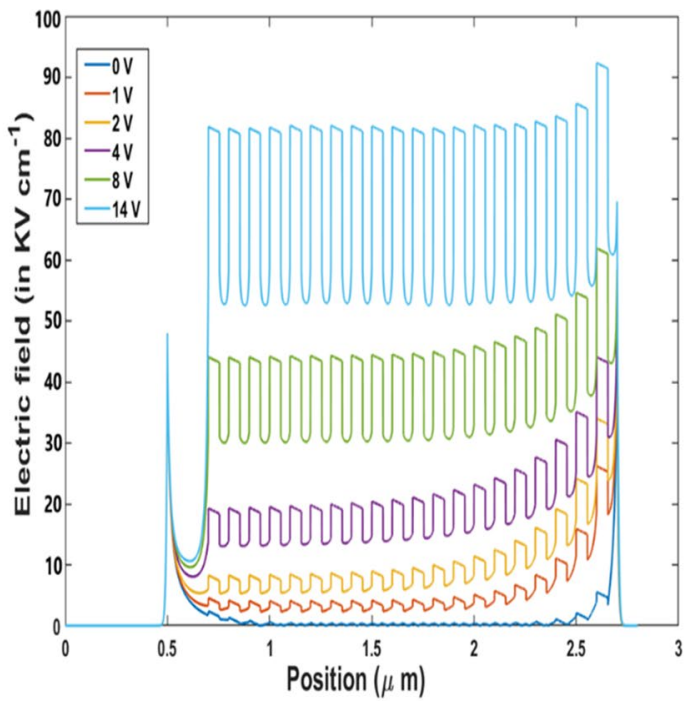

Figure 4. (a) Breakdown I-V characteristics and corresponding multiplication gain vs applied reverse bias at 200 K. Dark current and photocurrent are shown on the left axis; gain is shown on the right axis. (b) Simulated electric field profile under different applied reverse biases of SAM-APD structure.

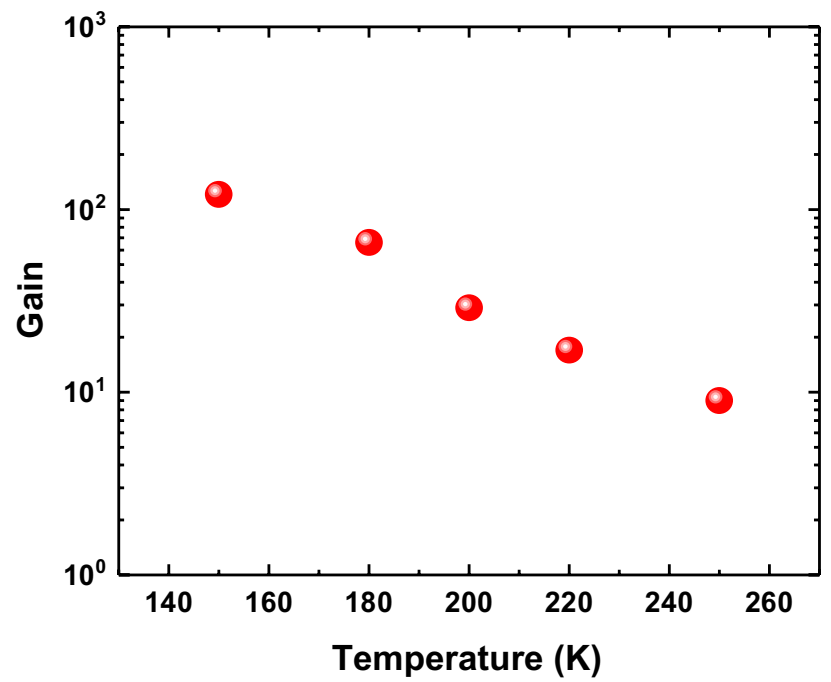

Figure 5. Temperature dependent gain characteristics of the InAsSb-based MWIR SAM-APD.

photocurrent ${ }^{28}$. The typical breakdown I-V characteristics of a $200 \times 200 \mu \mathrm{m}^{2}$ MWIR InAsSb APD is shown in Fig. 4(a). A multiplication gain around 29 was achieved at a reverse bias voltage of $-14.7 \mathrm{~V}$ at $200 \mathrm{~K}$. The unitygain current at $200 \mathrm{~K}$ is $6.6 \times 10^{-6} \mathrm{~A}$ at $0.9 \mathrm{~V}$. As shown in Fig. 4(a), the gain exhibits an exponential increase as a function of the reverse bias. This demonstrates that the InAsSb-based SAM-APD has the expected exponential multiplication gain characteristic which confirms single carrier electron-dominated impact ionization in the avalanche regime, as seen in other MWIR APDs ${ }^{29,30}$. Figure 4(b) shows the simulated electric field intensity distribution across the SAM-APD device structure, especially the multiplication region using a $1 \mathrm{D}$ finite element model $^{31}$. When the SAM-APD is under applied reverse bias, the electrons from the absorption region see the alternating strong and weak electric field in the MQW structure, as predicted theoretically in the previous part.

The relationship between the multiplication gain of the SAM-APD device and the temperature was also investigated as shown in Fig. 5. The temperature dependent gain characteristic exhibits the trend that the multiplication gain decreases continually from 121 to 10 while the temperature increases from 150 to $250 \mathrm{~K}$. At $150 \mathrm{~K}$, the maximum multiplication gain, around 121, for the MWIR SAM-APD device was achieved at $-16.8 \mathrm{~V}$ bias voltage. The multiplication gain value for this device is larger than the previous reports for III-V materials based MWIR APDs ${ }^{15}$. The decrease of the multiplication gain at higher temperature is because the different 


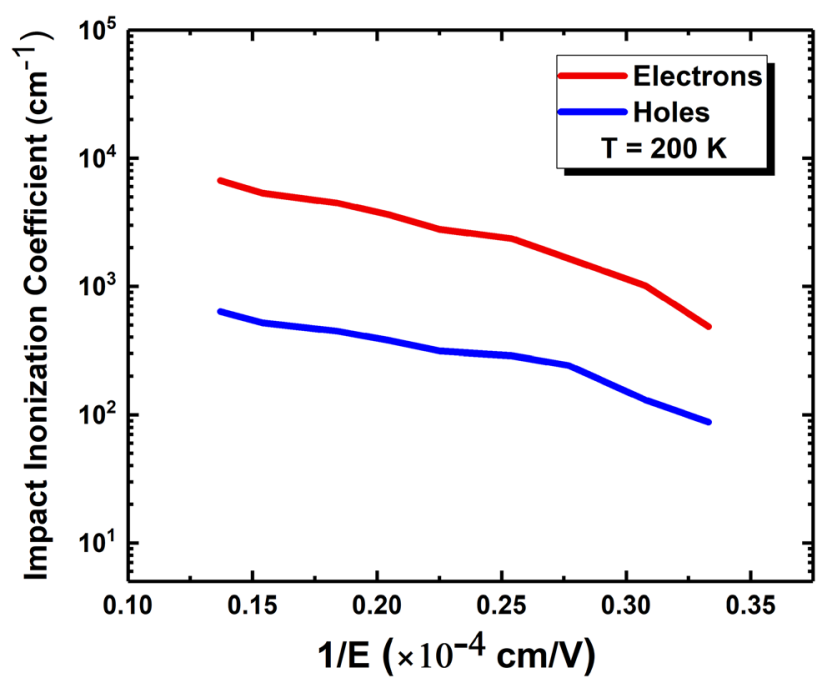

Figure 6. Electron and hole impact ionization coefficients for the MWIR SAM-APD vs inverse of electric field at $200 \mathrm{~K}$.

scattering mechanisms, such as lattice scattering and impurity scattering, become stronger when the temperature increases, making a higher loss of kinetic energy for carriers via scattering. It is more difficult for the carriers to reach the impact ionization threshold energy at higher temperature. In addition, carrier-carrier scattering is an important factor in semiconductor materials where the impact ionization process is significant. The higher probability of carrier-carrier scattering at higher temperature also leads to the decrease of the multiplication gain for the APD device ${ }^{32}$.

Another APD device with the flipped structure (the p-type contact at the top and the n-contact at the bottom) was grown and processed under the same conditions to derive the electron and hole impact ionization coefficients, $\alpha$ and $\beta$ from the experimental value of electron initiated avalanche gain $M_{e}$ and hole initiated avalanche gain $M_{h}$ by calculating the established formula ${ }^{33}$. The APD device with the flipped structure was illuminated from top p-type contact, where the carrier injection into the multiplication region is dominated by holes ${ }^{34}$. The derived electron and hole impact ionization coefficients are shown in Fig. 6, where the large difference between $\alpha$ and $\beta$ is seen. The carrier ionization ratio, $k$, defined as the ratio of hole to electron impact ionization coefficient, for the MWIR SAM-APD was calculated to be $\sim 0.097$ at $200 \mathrm{~K}$, which is smaller than the value of $k(0.27)$ achieved in the previous report ${ }^{15}$. This relatively small $k$ value is largely due to the enhanced electron impact ionization in MQW multiplication region, which also agrees well with other simulation and experiment results of this effect in similar structures like superlattices in the previous works ${ }^{24,35,36}$. The small carrier ionization ratio is essential for achieving low excess noise as demonstrated by McIntyre ${ }^{18}$. The carrier ionization ratio is still larger than the $k$ value $(\sim 0)$ achieved in InAs/GaSb superlattice based APD and can be further improved by tuning the band structure and loops of the MQW structure ${ }^{29}$.

In summary, the multi-quantum well structure using $\mathrm{AlAsSb} / \mathrm{GaSb} \mathrm{H}$-structure superlattice was incorporated into an InAsSb-based SAM-APD device as the multiplication region. The SAM-APD device was grown on GaSb substrate by $\mathrm{MBE}$ and designed to have electron-dominated avalanche mechanism by engineering the electron impact ionization rate in the MQW structure. The device exhibits a $100 \%$ cut-off wavelength of $\sim 4.6 \mu \mathrm{m}$ at $150 \mathrm{~K}$ and reaches the peak responsivity of $1.71 \mathrm{~A} / \mathrm{W}$ at $3.9 \mu \mathrm{m}$ under $-1.0 \mathrm{~V}$ applied bias. The multiplication gains of 29 were achieved for the SAM-APD device at $200 \mathrm{~K}$, respectively. The carrier ionization ratio was calculated to be 0.097 at $200 \mathrm{~K}$.

\section{Methods}

Growth. An Intevac Modular Gen II molecular beam epitaxy (MBE) equipped with group III SUMO cells and group V valved crackers was used to grow the SAM-APD structure on 2-inch Te-doped n-type $\left(10^{17} \mathrm{~cm}^{-3}\right)$ $\mathrm{GaSb}(100)$ substrate at $385^{\circ} \mathrm{C}$. The growth rates for InAsSb, AlAsSb and GaSb are determined by the growth rate of group III elements and are about $0.5 \mathrm{ML} / \mathrm{s}, 0.35 \mathrm{ML} / \mathrm{s}$ and $0.75 \mathrm{ML} / \mathrm{s}$, respectively. During growth, Silicon and Beryllium were used for n-type and p-type dopants, respectively.

Fabrication. After growth, the wafer was fabricated into the two-contact mesa-isolated devices with the sizes varying from $40 \times 40 \mu \mathrm{m}^{2}$ to $300 \times 300 \mu \mathrm{m}^{2}$ using our standard single element photodiode fabrication steps. The photolithography was first used to create the pattern of mesa shapes and sizes on the photoresist above the sample. Then the combination of inductively coupled plasma-reactive ion etching (ICP-RIE) and wet etching was used to transfer the pattern of the photomask onto the sample to define the shape of the mesa. The wet etching right after ICP-RIE can remove the residue and smoothen the sidewall, which can reduce the surface leakage current. All the devices were cleaned thoroughly during processing to minimize the dark current. A second photolithography was used to define the top and bottom contact areas. Then the top and bottom metal 
contacts consisted of $\mathrm{Ti} / \mathrm{Au}$ were deposited via electron beam metal evaporation. $\mathrm{A} \mathrm{SiO}_{2}$ layer was deposited on the sample via plasma enhanced chemical vapor deposition (PECVD) to passivate the devices. The window to the metal contacts was opened by performing another photolithography and removing the $\mathrm{SiO}_{2}$ by reactive ion etching. The metal contacts for wire-bonding were then deposited.

Device testing. The SAM-APD test chip was mounted onto a 68-pin leadless chip carrier (LCC) and then loaded into a Janis STVP-100 two chamber liquid helium cryostat station with controlled temperature ranging from 150 to $200 \mathrm{~K}$. The relative spectral response of the InAsSb-based MWIR SAM-APD was measured at both $150 \mathrm{~K}$ and $200 \mathrm{~K}$ under front-side illumination using a Bruker IFS 66v/S Fourier transform infrared spectrometer (FTIR). A calibrated blackbody source at $1000{ }^{\circ} \mathrm{C}$ was then used to calculate the absolute optical responsivity of the photodiodes.

Current-voltage (I-V) measurements of the SAM-APD sample were carried utilizing an Agilent 4156c semiconductor parameter analyzer. A $633 \mathrm{~nm} \mathrm{He}-\mathrm{Ne}$ laser with an incident power of $5.0 \mathrm{~mW}$ was used to measure the photocurrent and the gain of the APDs at temperature range from 150 to $250 \mathrm{~K}$.

Received: 28 December 2020; Accepted: 15 March 2021

Published online: 29 March 2021

\section{References}

1. M. Razeghi, Sb-based third generation at Center for Quantum Devices (SPIE Defense + Commercial Sensing). SPIE, 2020.

2. M. Razeghi et al., Antimonite-based gap-engineered type-II superlattice materials grown by MBE and MOCVD for the third generation of infrared imagers (SPIE Defense + Commercial Sensing). SPIE, 2019.

3. Li, J., Dehzangi, A., Wu, D., McClintock, R. \& Razeghi, M. Type-II superlattice-based heterojunction phototransistors for high speed applications. Infrared Phys. Tech. 108, 103350. https://doi.org/10.1016/j.infrared.2020.103350 (2020).

4. Dehzangi, A. et al. Extended short wavelength infrared heterojunction phototransistors based on type II superlattices (in English). Appl. Phys. Lett. 114(19), 1 (2019).

5. Campbell, J. C., Dentai, A. G., Holden, W. S. \& Kasper, B. L. High-performance avalanche photodiode with separate absorption 'grading' and multiplication regions. Electron. Lett. 19(20), 818-820. https://doi.org/10.1049/el_19830558 (1983).

6. Chuang, L. C. et al. GaAs-based nanoneedle light emitting diode and avalanche photodiode monolithically integrated on a silicon substrate. Nano Lett. 11(2), 385-390. https://doi.org/10.1021/nl102988w (2011).

7. Kerlain, A. et al. Performance of mid-wave infrared HgCdTe e-avalanche photodiodes. J. Electr. Mater. 41(10), 2943-2948. https:// doi.org/10.1007/s11664-012-2087-5 (2012).

8. Sun, X. et al. HgCdTe avalanche photodiode detectors for airborne and spaceborne lidar at infrared wavelengths. Opt. Express 25(14), 16589-16602. https://doi.org/10.1364/OE.25.016589 (2017).

9. Rogalski, A. HgCdTe infrared detector material: history, status and outlook. Rep. Progr. Phys. 68(10), 2267 (2005).

10. P. Castelein et al., Megapixel HgCdTe MWIR focal plane array with a 15-um pitch (AeroSense 2003). SPIE, 2003.

11. B.-M. Nguyen, Theoretical Design and Material Growth of Type-II Antimonide-based Superlattices for Infrared Detection and Imaging. 01/01 2010.

12. Razeghi, M. InAs/GaSb type II superlattices: a developing material system for third generation of IR imaging. In Mid-infrared Optoelectronics (eds Tournié, E. \& Cerutti, L.) (Woodhead Publishing Press, 2020).

13. Haddadi, A., Dehzangi, A., Adhikary, S., Chevallier, R. \& Razeghi, M. Background-limited long wavelength infrared InAs/InAs1xSbx type-II superlattice-based photodetectors operating at $110 \mathrm{~K}$. APL Mater. 5(3), 035502. https://doi.org/10.1063/1.4975619 (2017).

14. Li, J., Dehzangi, A., Wu, D., McClintock, R. \& Razeghi, M. Resonant cavity enhanced heterojunction phototransistors based on Type-II superlattices. Infrared Phys. Tech. 1, 103552. https://doi.org/10.1016/j.infrared.2020.103552 (2020).

15. Dehzangi, A., Li, J., Gautam, L. \& Razeghi, M. Avalanche Photodetector Based on InAs/InSb Superlattice. Quantum Rep. 2(4), 591-599 (2020).

16. Carrano, J. C. et al. GaN avalanche photodiodes. Appl. Phys. Lett. 76(7), 924-926. https://doi.org/10.1063/1.125631 (2000).

17. Pau, J. L., Bayram, C., McClintock, R., Razeghi, M. \& Silversmith, D. Back-illuminated separate absorption and multiplication GaN avalanche photodiodes. Appl. Phys. Lett. 92(10), 101120. https://doi.org/10.1063/1.2897039 (2008).

18. McIntyre, R. J. Multiplication noise in uniform avalanche diodes. IEEE Trans. Electron Dev. 13(1), 164-168. https://doi.org/10. 1109/T-ED.1966.15651 (1966).

19. Haddadi, A., Chevallier, R., Dehzangi, A. \& Razeghi, M. Extended short-wavelength infrared nBn photodetectors based on typeII InAs/AlSb/GaSb superlattices with an AlAsSb/GaSb superlattice barrier. Appl. Phys. Lett. 110(10), 101104. https://doi.org/10. 1063/1.4978378 (2017).

20. Nguyen, B.-M. et al. Band edge tunability of M-structure for heterojunction design in Sb based type II superlattice photodiodes. Appl. Phys. Lett. 93(16), 163502. https://doi.org/10.1063/1.3005196 (2008).

21. Wei, Y. \& Razeghi, M. Modeling of type-II InAs/GaSb superlattices using an empirical tight-binding method and interface engineering. Phys. Rev. B 69(8), 085316. https://doi.org/10.1103/PhysRevB.69.085316 (2004).

22. Schuler-Sandy, T. et al. Growth of InAs-InAsSb SLS through the use of digital alloys. J. Cryst. Growth 425, 29-32. https://doi.org/ 10.1016/j.jcrysgro.2015.02.096 (2015).

23. Ren, M. et al. AlInAsSb/GaSb staircase avalanche photodiode. Appl. Phys. Lett. 108(8), 081101. https://doi.org/10.1063/1.49423 70 (2016).

24. Capasso, F., Tsang, W. T., Hutchinson, A. L. \& Williams, G. F. Enhancement of electron impact ionization in a superlattice: A new avalanche photodiode with a large ionization rate ratio. Appl. Phys. Lett. 40(1), 38-40. https://doi.org/10.1063/1.92910 (1982).

25. Wu, D., Li, J., Dehzangi, A. \& Razeghi, M. Mid-wavelength infrared high operating temperature pBn photodetectors based on type-II InAs/InAsSb superlattice. AIP Adv. 10(2), 025018. https://doi.org/10.1063/1.5136501 (2020).

26. Haddadi, A., Dehzangi, A., Chevallier, R., Adhikary, S. \& Razeghi, M. Bias-selectable nBn dual-band long-/very long-wavelength infrared photodetectors based on InAs/InAs1-xSbx/AlAs1-xSbx type-II superlattices. Sci. Rep. 7(1), 3379. https://doi.org/10. 1038/s41598-017-03238-2 (2017).

27. J. Li, A. Dehzangi, D. Wu, and M. Razeghi, High speed short wavelength infrared heterojunction phototransistors based on type II superlattices (SPIE OPTO). SPIE, 2020.

28. Bayram, C. et al. High quantum efficiency back-illuminated GaN avalanche photodiodes. Appl. Phys. Lett. 93(21), 211107. https:// doi.org/10.1063/1.3039061 (2008).

29. Mallick, S. et al. Ultralow noise midwave infrared InAs-GaSb strain layer superlattice avalanche photodiode. Appl. Phys. Lett. 91(24), 241111. https://doi.org/10.1063/1.2817608 (2007). 
30. Kinch, M., Beck, J., Wan, C.-F., Ma, F. \& Campbell, J. HgCdTe electron avalanche photodiodes. J. Electr. Mater. 33(6), 630-639 (2004).

31. Winston, D. \& Hayes, R. SimWindows-a new simulator for studying quantum-well optoelectronic devices. Compd. Semicond. 1994(141), 747-750 (1995).

32. Binder, R. et al. Carrier-carrier scattering and optical dephasing in highly excited semiconductors. Phys. Rev. B 45(3), 1107-1115. https://doi.org/10.1103/PhysRevB.45.1107 (1992).

33. Lee, C. A., Kleimack, J. J., Batdorf, R. L., Wiegmann, W. \& Logan, R. A. Ionization Rates of Holes + Electrons in Silicon (in English). Phys. Rev. 134(3a), A761. https://doi.org/10.1103/PhysRev.134.A761 (1964).

34. Oguzman, I. H. et al. "Theory of hole initiated impact ionization in bulk zincblende and wurtzite GaN," (in English). J. Appl. Phys. 81(12), 7827-7834. https://doi.org/10.1063/1.365392 (1997).

35. Brennan, K. Theory of electron and hole impact ionization in quantum well and staircase superlattice avalanche photodiode structures. IEEE Trans. Electron Devices 32(11), 2197-2205. https://doi.org/10.1109/T-ED.1985.22258 (1985).

36. Aristin, P., Torabi, A., Garrison, A. K., Harris, H. M. \& Summers, C. J. New doped multiple-quantum-well avalanche photodiode: the doped barrier Al0.35Ga0.65As/GaAs multiple-quantum-well avalanche photodiode. Appl. Phys. Lett. 60(1), 85-87. https:// doi.org/10.1063/1.107383 (1992).

\title{
Acknowledgments
}

This work was partially supported by the Defense Advanced Research Projects Agency (DARPA) under agreement number FA8650-18-1-7810. Authors would like to acknowledge the support and encouragement of Dr. Whitney Mason from DARPA., support and encouragement of Dr. Michael Gerhold and Dr. Tania Paskova from the U.S. Army Futures Command, Dr. Kurt Eyink from the Air Force Research Laboratory, Dr. Murzy Jhabvala from NASA Goddard Space Flight Center, and Dr. Meimei Tidrow from the U.S. Army Night Vision, Laboratory.

\section{Author contributions}

J.L. conducted the MBE growth, performed the characterization and modelling, and wrote the paper. A.D. conducted device fabrication and calculated the multiplication gain. G.B. revised and modified the manuscript. M.R. provided the idea and supervised the project. All authors reviewed the manuscript.

\section{Competing interests}

The authors declare no competing interests.

\section{Additional information}

Correspondence and requests for materials should be addressed to M.R.

Reprints and permissions information is available at www.nature.com/reprints.

Publisher's note Springer Nature remains neutral with regard to jurisdictional claims in published maps and institutional affiliations.

\begin{abstract}
(c) (1) Open Access This article is licensed under a Creative Commons Attribution 4.0 International License, which permits use, sharing, adaptation, distribution and reproduction in any medium or format, as long as you give appropriate credit to the original author(s) and the source, provide a link to the Creative Commons licence, and indicate if changes were made. The images or other third party material in this article are included in the article's Creative Commons licence, unless indicated otherwise in a credit line to the material. If material is not included in the article's Creative Commons licence and your intended use is not permitted by statutory regulation or exceeds the permitted use, you will need to obtain permission directly from the copyright holder. To view a copy of this licence, visit http://creativecommons.org/licenses/by/4.0/.
\end{abstract}

(C) The Author(s) 2021 